\title{
EL SISTEMA DE LA GRADACIÓN EN LATÍN: NOCIÓN BÁSICA, ESTRUCTURA Y USOS
}

In front of the traditional and modern theories which see two grammatical categories in the latin gradation, we shall suggest in this paper the existence of just one grammatical category, whose basic notion is not the idea of comparison but that of elation. In the light of this assumption, we shall propose a structure which permits us to explain satisfactorily all the uses of the degrees, even those which seem to be anomalous.

\section{I. ¿UNO O DOS SISTEMAS DE OPOSICIONES?}

La tradición escolar señala que el adjetivo latino puede adoptar, además del positivo, los grados comparativo y superlativo, y que cada uno de ellos puede ser, a su vez, absoluto o relativo. Los absolutos reciben la denominación específica de intensivo y elativo, respectivamente; y en cuanto a los relativos, añade esa tradición que si la comparación se establece entre dos elementos se emplea el grado comparativo (doctior fratre) y que si se establece entre más, se usa el superlativo (doctissimus omnium) '.

Pues bien, en este doble uso de los grados se ha intentado ver la posibilidad de un nuevo análisis, como si en la gradación hubiera no una, sino dos categorías gramaticales. Asi procede $\mathrm{H}$. Fugier ${ }^{2}$, quien asegura que en latín no existen dos comparativos (absoluto -o intensivo- y relativo) y dos superlativos (absoluto - o elativo- y relativo), sino un «cuantificador», representado por los tradicionales grados intensivo y elativo, que se caracteriza por no admitir nunca complemento, y dos «clasificadores» caracterizados ambos por recibir siempre complemento, pero distintos en razón del distinto complemento que uno y otro reciben.

E1 «clasificador 1» de Fugier sería el tradicional superlativo rełativo que se construye con un complemento en genitivo (doctissimus omnium) o preposi-

\footnotetext{
' Vid., por ejemplo, J.B. Hofmann-A. Szantyr 1972, p. 162; o M. Bassols de Climent, 1950-52 p. 188 s. y 1956, p. 165; A. Ernout-F. Thomas 1972, pp. 167 s.

${ }^{2}$ Fugier 1972, passim, esp. pp. 272-281.
} 
cional del tipo inter + acusativo (doctissimus inter omnes); un complemento esperable, toda vez que implica la inclusión del sustantivo a que el clasificador se refiere en la serie de referencia que se expresa en él, bien porque, en el caso del genitivo, éste es partitivo y expresa el todo del que aquel sustantivo es parte, bien porque en el giro preposicional se indica léxicamente (inter) esa inclusión.

El «clasificador 2 », naturalmente, sería el tradicional comparativo relativo. Su construcción con quam (doctior quam fratres), pero sobre todo con ablativo (doctior fratribus), indica que el sustantivo a que se refiere el adjetivo queda excluido de la serie de elementos que se presentan en el «segundo término» de la comparación.

Ahora bien, aunque podamos admitir que, como Fugier dice, unos mismos significantes posean dos significaciones distintas (cuantificador y clasificadores) ${ }^{3}$, dificilmente podemos compartir, en cambio, las tesis de que, por una parte, los valores de los grados se definan por algo extraño a ellos, como lo es el que reciban o no complementación, y, por otra parte, de que, en el caso del cuantificador, a sus dos significantes distintos corresponda una misma significación; al contrario, estimamos que si hay un «cuantificador en -ior» y un «cuantificador en -issimus», como ella los llama, hay dos cuantificadores $-\mathrm{y}$ no uno-, por muy estrecha coherencia que los vincule ${ }^{4}$ y por muy unitarios que parezcan al no recibir complementación alguna.

También por dos oposiciones distintas se decide en su análisis J. A. Correa, más ajustado al tenor de la gramática tradicional: una de comparación y otra de intensidad ${ }^{5}$. A su entender, la de comparación es sintáctica, porque, además de los sufijos que caracterizan a sus miembros, uno y otro precisan complementación; si la comparación se establece entre dos términos, aparece el grado comparativo, como en maior natu tratándose, por ejemplo, de dos hermanos; si entre más de dos términos, el grado superlativo, como en maximus natu. Por su parte, la oposición de intensidad es de carácter semántico y se caracteriza, como el cuantificador de Fugier, por carecer de complemento.

Pero esa diversidad de valores que se pretende apreciar en la categoría está, en nuestra opinión, poco justificada ${ }^{6}$. En efecto, como subraya M. Poirier a propósito del superlativo ${ }^{7}$, ni morfológicamente se muestran diferencias sig-

\footnotetext{
${ }^{3}$ Fugier 1972, p. 274.

4 Fugier 1972, p. 287.

${ }^{5}$ Correa 1978 , pp. 535 s.

6 No nos parece, pues, que la cuestión esté, como asegura C. Arias Abellán 1984, p. 125, «definitivamente aclarada».

${ }^{7}$ M. Poirier 1991, «Superlatif relatif et superlatif absolu. Cette distinction est-elle incontcstable?», Comunicación presentada en el VI Colloquio de Lingüística Latina, Budapest, 23-27 de abril. El autor nos envió gentilmente su original.
} 
nificativas de valores absolutos o relativos en la forma -issimus, ni puede considerarse que, en lo que afecta al superlativo relativo, haya una especie de «morfema discontinuo» representado por aquella forma -issimus y el complemento en genitivo o preposicional, ya que muchas veces está ausente. Así, en:

- Cic., Cat. II 3 Interfectum esse L. Catilinam et grauissimo supplicio affectum iampridem oportebat;

- Cic., Cat. III 23 Erepti enim estis ex crudelissimo ac miserrimo interitu,

los pasajes podrían ser interpretados como ejemplos de cualquiera de las dos acepciones: la relativa, sobreentendiendo fácilmente, por obvio, un complemento del tipo omnium suppliciorum / omnium interituum, o la absoluta. Porque, en realidad, ni una ni otra acepción son propias del superlativo, sino que más bien parecen responder a sendos efectos de sentido que aquél adopta en la frase. Sólo hay, pues, un superlativo, que puede ser absoluto o relativo, según el contexto.

Otro tanto nos parece que cabe decir del grado comparativo. Ni de la morfología de doctior se deducen, como es natural, dos categorías, ni de la presencia o ausencia del llamado segundo término de la comparación nos parece que quepa inferirlas: hay contextos en que esa presencia revela un sentido inequívocamente comparativo de la frase (no del adjetivo), pero también otros en que, sin estar presente el complemento, no podemos decidir si la estructura es comparativa o intensiva ${ }^{8}$. Es lo que sucede en este pasaje de Cicerón:

- Cic., Cato mai. 41 Si quidem ea (sc. uoluptas), cum maior esset atque longior, omne animi lumen exstingueret;

donde R. Kühner-C. Stegmann ${ }^{9}$ añaden: «(sc. quam decet)». O en este de Plauto:

- Pl., Epid. 10 Corpulentior uidere atque habitior,

donde puede interpretarse que «me pareces bastante corpulento y robusto», con sentido intensivo, o «me pareces más corpulento y robusto» (sc. «de lo que estabas»), con idea comparativa. O en este otro de Catón, típicamente catalogado como intensivo,

- Cat., $A g r .55$, senectus est natura loquacior,

${ }^{8}$ Cf. Arias Abellán 1984, p. 128: «Siempre que nos ha sido posible reconocerle un segundo término de comparación (aunque no estuviese expreso en los textos), hemos dado al morfema -ior una función estrictamente comparativa y no cuantificadora. Desde este punto de mira, son realmente escasos los usos clasificables, con absoluta seguridad como I(ntensivos)) .

${ }^{9}$ R. Kühner-C. Stegmann 1914, II 2, p. 476. 
pero que también puede entenderse como comparativo relativo con su complemento más que esperable implícito: quam iuventus o iuventute o, también, quam decet.

Sólo hay, pues, u n comparativo que, como el superlativo, puede ser absoluto o relativo según el contexto. Por ello, parece más apropiado considerar que en la gradación hay una sola categoría gramatical, y no dos.

\section{NOCIÓN Y ESTRUCTURA DE LA CATEGORÍA}

Dado que, entonces, forman la categoria sólo los términos doctus, doctior y doctissimus, debemos definir en qué consiste la noción básica de «grado» que se atribuye a doctior y doctissimus frente a doctus y que, según es común admitir, éste no posee.

Lo primero que podemos decir es que esa noción no es la de «comparación». En efecto, sólo puede hablarse de comparación cuando en el contexto existe o se sobreentiende un término de referencia, el llamado «segundo término de la comparación», o, lo que es lo mismo, cuando el comparativo o superlativo tienen sentido relativo. Ahora bien, como el sentido relativo de comparativo y superlativo es, según decíamos, contextual, la comparación misma no puede ser su noción básica; en realidad, los tradicionalmente llamados grados de comparación del adjetivo no comparan.

En cambio, sí nos parece que la idea de intensidad, que subyace en la otra pretendida oposición de grado, se ajusta más a la noción básica que buscamọ; pues, en efecto, en su descripción se señala que, descartado un segundo término que adscriba al adjetivo a la oposición comparativa, unas formas se oponen a otras en virtud de una mayor o máxima intensidad de la idea expresada por el adjetivo.

Pues bien, estimamos que precisamente en esa mayor o máxima intensidad de la idea que expresa el adjetivo en grado, a la que llamaremos el a c ión, reside la noción básica de la categoría; una noción semántica que poseen doctior y doctissimus paradigmáticamente frente a doctus, con total independencia del contexto 10 y que, por su significado de «elevación», responde, además, al sentido positivo consustancial de la categoría, esto es, al de elación, por así decirlo, de «signo más», no de «signo menos»: la gradación adjetiva de inferioridad es, en latín, léxica y, por lo tanto, no gramatical, de la misma manera que la de superioridad con magis tampoco lo es. Sólo la expresión de los grados de superioridad por medio de los morfemas -ior/-issimus es gramatical ".

\footnotetext{
${ }^{10}$ La noción, por tanto, se revela como contintadora del original valor que las formas indoeuropeas *io:s/-ios, *-eros/-teros poselan. Vid. A. Díaz Tejera 1985, p. 279 s.: use empleó para marcar la intensidad y evaluación de la noción básica a la que se añadía» (279).

11 Por eso no estamos de acuerdo en que se incluya su estudio en el de los grados del
} 
Ahora bien, si los grados del adjetivo se definen paradigmáticamente por las relaciones que entre sí mantienen, ¿de dónde sale el supuesto valor comparativo que se atribuye a doctior y doctissimus? La respuesta es sencilla: de sus relaciones sintagmáticas con el enunciado: el adjetivo en grado, sin perder su valor propio paradigmático, queda a disposición del contexto para contribuir a significar una comparación ${ }^{12}$. Es lo mismo que sucede con las «comparaciones de igualdad y diferencia» ${ }^{13}$ expresadas por idem, alius, pariter, simul, etcétera: la idea de igualdad o diferencia está en el lexema del pronombre o adverbio, mas no la de comparación que, en cambio, se deduce del contexto $\mathrm{y}$, en particular, de la construcción con $a c$ y otro término. Así puede explicarse la ambigüedad de la que habla Correa cuando afirma que «la comparación no es imprescindible que vaya explicitada», lo que hace que «en determinados contextos ambas oposiciones se neutralicen entre si, quedando al hablante u oyente la posibilidad de entenderlas en cualquiera de los dos sentidos o incluso en ambos a la vez» ${ }^{14}$; porque si, como hemos dicho, no hay dos oposiciones, sino una, no puede haber neutralización entre ellas ${ }^{15}$; y si lo propio de los grados del adjetivo no es indicar comparación, sino elación, lo natural es que tal comparación no requiera ser explicitada.

Queda ahora por averiguar lo que distingue a doctior y doctissimus entre sí. Frente a la indiferencia que muestra doctior respecto a si esa elación es la máxima posible o no, doctissimus la significa, es decir, significa la elación en grado superelativo ${ }^{16}$. Claro está que esa elación superelativa es la máxima que gramaticalmente puede reflejar el latín, lo que no obsta para que haya contex-

adjetivo, como parte de su paradigma de «cuantificación», como hace Fugier 1972, pp. 287 ss.; ni, por supuesto, junto a la forma léxica de indicar superioridad (ibid.). Si, en cambio, pueden estudiarse esos procedimientos, junto con las derivaciones y prefijaciones, cuando se trata de exponer los recursos por medio de los cuales se consigue añadir intensidad, como hace Arias Abellán 1984 y 1987.

${ }^{2}$ Según Díaz Tejera 1985, en cambio, «estos elementos adquieren función categorial de comparativos» (p. 280); «el valor comparativo de estos lexemas surge por un cambio de sentido posicional hacia cualitativo y por su construcción sintáctica» (p. 284). Lo más exacto sería decir que, en la distribución de la que él habla de morfemas de comparación y superlación, unos quedaron a merced de la frase para expresar nociones comparativas, sin poseerlas, los que se aplicaban a lexcmas cualitativos, y otros no, los aplicados a lexemas espacio-temporales. Eso encaja muy bien con su afirmación de que el sufijo -mus/timus «nunca fue, en latín, un morfema de superlativo» (p. 287).

13 Ernout-Thomas 1972, pp. 173 s.

14 Conrea 1978, p. 539.

15 Lo que, por otra parte, es dificil de entender; la neutralización parece más comprensible tratándose de términos de una oposición, pero no entre dos oposiciones distintas.

16 Tal como, en otros téminos, sugiere Poirier 1991, quien opina que la única signilicación del superlativo viene a ser la de representar «el grado terminal de la escala de la cualidad expresada por el adjetivo». 
tos y giros cuyo significado sobrepase ese valor; pero su producción no es gramatical. Así sucede, por ejemplo, en el conocido pasaje de Cicerón:

- Cic., Off. III 121 Vale igitur, mi Cicero, tibique persuade esse te quidem carissimum sed multo fore cariorem si talibus monumentis praeceptisque laetabere ${ }^{17}$;

donde cariorem, aun sin el refuerzo de multo, expresa un grado de afecto mayor incluso que el señalado por carissimum: el contexto coloca, por así decirlo, el listón del afecto en lo más alto por medio del superlativo y, a partir de ahí, la elación de cariorem permite sobrepasarlo.

Sabidos, pues, los valores reales que, en nuestra opinión, poseen los grados latinos, podemos intentar una estructuración de la categoría. Por de pronto, ya tenemos un término que podemos considerar como no marcado en relación con el valor elativo: doctus. Pero su definición exacta no es, entonces, la de "grado positivo», sino la de término o grado no elativo, pues lo que lo caracteriza es justamente no poseer elación: es el término o grado no marcado.

Por lo que se refiere a los otros dos términos, es claro, según lo dicho, que si ambos poseen elación y uno de ellos la posee en el grado más elevado posible, el «grado super», este ha de ser más marcado que el otro. De forma tal que la definición exacta de doctior es la de término o grado elativo y no superelativo, en tanto que la de doctissimus es la de término o grado elativo y superelativo.

Ahora bien, definidos así los grados, siempre será más cómodo usar las denominaciones tradicionales de la misma manera que las usamos en la categoría del género al referimos al neutro, masculino y femenino, cuando, en realidad, esos términos se definen exacta y respectivamente como «no animado», «animado no femenino» y «animado femenino». En nuestro caso, para tres solos términos disponemos de cinco nombres acuñados por la tradición: positivo, comparativo, intensivo, superlativo y elativo. Nada objetaremos al nombre «positivo» para doctus, si por él se entiende valor de grado no marcado, siendo así que es común en ambas pretendidas series de sentidos, la relativa y la absoluta; mas no nos parece muy adecuado el de «comparativo» para doctior, porque expresa justamente el valor del que, según tratábamos de demostrar, carece el término: para esa forma preferimos «intensivo». Y como «elativo» ya lo hemos usado para definir con más propiedad la noción básica de la categoría, nos quedamos con «superlativo» para doctissimus, un nombre que le va de maravilla si es que refleja la «elación super» o máxima.

Gráficamente, y según lo expuesto, la categoría se organizaría asi 18 :

17 Vid. Poirier 1991 y Correa 1978, p. 537.

18 De modo similar, vid. Arias Abellán 1984, p. 136 y 1987, nota 12. Sin embargo, en su interpretación sigue adoptando el análisis propuesto por Correa 1978, de ver dos oposi- 


$$
\frac{\text { doctus }(\varnothing)}{\text { doctior }(+1) / \text { doctissimus }(+1,+2)}
$$

$+1=$ elación

$+2=$ superelación

o bien:

$$
\frac{\text { positivo }(\varnothing)}{\text { intensivo }(+1) / \text { superlativo }(+1,+2)}
$$

Como se ve, se establecen dos oposiciones privativas en las que los términos menos marcados habrán de tener la propiedad de poder aparecer, además de como lo que son, con el valor de los respectivos términos marcados; y habrá de cumplirse la condición de la «no reversibilidad de los términos», es decir, de que los marcados no puedan aparecer por los no marcados ${ }^{19}$.

\section{Usos: maior natu/maximus natu}

El anterior análisis choca frontalmente con el propuesto por Correa, también estructural. En efecto, estima este autor que de los dos términos marcados frente al positivo, el comparativo es más marcado que el superlativo, ya que para que sea posible su presencia la comparación ha de establecerse entre dos conceptos, condición que no exige el superlativo. Y así parece cumplirse en los clásicos ejemplos: maior natu, tratándose de dos hermanos, pero maximus natu, si son más de dos hermanos. Que aparezca maximus natu cuando se habla de dos hermanos, sería un uso neutro del «superlativo» por el «comparativo» que parece corroborar el análisis de Correa ${ }^{20}$.

Pero las apariencias engañan. Por de pronto, si decíamos que la idea de comparación no es lo que caracteriza la oposición, con más dificultad puede admitirse que lo que opone a doctior frente a doctissimus sea que aquél «compare» entre dos y éste entre más de dos. ¿Cómo se explica, entonces, esa regular evidencia de que cuando son dos los términos sintagmáticamente comparados aparece el «comparativo» (nuestro intensivo) y de que cuando son más de dos el superlativo? Muy fácilmente: cuando los términos son dos, decir de uno de ellos que posee elación equivale a decir que posee superelación, por lo

ciones; sólo la de intensidad se organizaría de esta manera. En su estructura, además, incluye una oposición privativa entre el intensivo no absoluto (el tradicional grado intensivo, frente al intensivo absoluto, el tradicional elativo) y un intensivo no absoluto con " "marca del término de relación" satis, nimis», que parece responder al giro analítico compuesto por esos adverbios y el positivo. Esto último no nos parece oportuno, por cuanto supone mezclar, como hemos subrayado, lo puramente gramatical con lo léxico, por muy equivalentes que semánticamente parezcan ser.

19 Vid. M. S. Ruipérez 1954 , pp. 16 ss.

20 Correa 1978 , p. 536. 
menos respecto al otro. O yendo al caso de los dos hermanos, decir que uno es mayor que el otro es lo mismo que decir que es «el mayor»; de ahí que suela aparecer el grado intensivo: que el resultado sea que equivale a un superlativo es un efecto de sentido propiciado por el contexto. O dicho en términos más gramaticales, es un uso neutro del intensivo por el superlativo. Vistas así las cosas, el uso del superlativo en casos como los anteriores no ha de extrañarnos tampoco, pues aparece con su legítimo valor de superelación o elación máxima ${ }^{21}$, y no, por supuesto, en lugar del grado comparativo, como afirmaba Correa.

Por lo demás, y de paso, se entiende ahora muy bien que, «teniendo en cuenta que el hablante puede siempre reducir, en principio, la comparación en una serie de términos a sólo dos, el primero y todo lo demás» ${ }^{22}$, aparezca el «comparativo» donde esperaríamos, por aparecer una serie de elementos en el segundo término, el superlativo: el efecto de sentido contextual es de superlativo (doctior fratribus $=$ doctissimus fratrum) por la misma razón que maior natu, entre dos, equivale a maximus natu: uso neutro de intensivo por superlativo. La diferencia está en la presentación sintagmática: el intensivo provoca la exclusión del término a que se refiere de la serie expresada en el «segundo término», mediante la construcción de éste en ablativo; el superlativo su inclusión, mediante el uso del genitivo o giro preposicional con inter.

\section{Doctior quam prudentior Y OTROS Usos}

En expresiones como doctior quam prudentior se dice que cabría esperar el grado no marcado, o grado positivo, en el segundo término ${ }^{23}$. Se han ofrecido varias explicaciones. En opinión de Fugier, la razón de que aparezca el «comparativo» estriba en que, no tratándose de dos personas, sino de una sola de quien se comparan dos cualidades, lo que se oculta en la construcción bajo una forma ramassée son «dos proposiciones distintas comparadas entre ellas, de las que cada una contiene a su vez una comparación de tipo simple» (...) «lo que provoca la simetría de las dos formas en -ior ... es pues un embottement antes que una reciprocidad» ${ }^{24}$.

21 Desde luego, no nos parece un uso neutro de «superlativo» por «comparativo» el alegado por Arias Abellán 1984, p. 126, de Plauto Amph. 548, atque quanto, nox, fuisti longior hac proxuma, por cuanto proxuma aparece con valor superelativo, en referencia a la «noche última», «la más cercana». Por lo demás, proxuma pertenece a ese elenco de formas con valor espacio-temporal a las que originariamente se aplicaron morfemas que luego, extendidos a adjetivos y adverbios de cualidad, se convirtieron en superlativos.

22 Correa 1978, pp. 535 s.

23 Vid., por ejemplo, Bassols 1956, p. 166.

24 Fugier 1972, p. 286. 
De semejante parecer es E. Sánchez Salor, quien estima que en construcciones de ese tipo el segundo adjetivo aparece en grado «comparativo» para facilitar la comprensión del primero con su doble función de «primer término» de la comparación y "clasificador»; ello permite comprender que prudentior sea «el segundo término de una comparación, el primero de los cuales tiene la misma forma» (...) «se trata de un tipo de comparación... en que hay equifuncionalidad entre el primero y el segundo término, exigiéndose por tanto cierta identidad formal» ${ }^{25}$. Esa equifuncionalidad parece responder, según Sánchez Salor, a que en esas comparaciones no hay una, sino dos predicaciones.

Y, por su parte, Correa, considera que en prudentior no hay uso del «comparativo» por el positivo, sino «una extensión mecánica de la forma del primer elemento al segundo, que está en el mismo caso y que no admite posibilidad de gradación; prueba de ello es que no se da el tipo magis prudens quam doction? ${ }^{26}$. Concluye Correa que ahí se produce una neutralización de la oposición.

Desde nuestro punto de vista, y teniendo en cuenta que la noción básica de la categoría no es la de comparación, sino la de elación, que aparezca prudentior en el segundo término de una comparación entre cualidades sólo requeriría explicación si, efectivamente, su valor fuera el de prudens, porque entonces estaríamos ante una notable anomalía en la que un término marcado aparecería por el no marcado.

Pero estimamos que no hay nada de ello. Si, como reconoce Fugier, la comparación que se establece en estas estructuras consiste «en medir la intensidad relativa de dos cualidades» ${ }^{27}$, la elación o intensidad habrá de existir en cada una de esas dos cualidades, razón por la que el grado intensivo se justifica en uno y otro adjetivos.

Por otra parte, esa «identidad formal» a que alude Sánchez Salor, exigida por la «equifuncionalidad» existente entre los términos de las «dos predicaciones», sostenidas también por Fugier, se explica muy bien si se piensa en que el grado intensivo de prudentior se amolda como anillo al dedo a las exigencias de la coordinación latina; porque estimamos que quam es una conjunción coordinante que, como tal, une términos isofuncionales, reuniendo en una lo que podrían ser predicaciones distintas. De hecho, puede conmutar, en determinadas condiciones, por $a c^{28}$ :

\footnotetext{
25 Sánchez Salor 1980, p. 223.

26 Correa 1978, p. 537.

27 Fugier 1972, p. 286, nota 1.

28 Además, por supuesto, de aparecer en estructuras comparativas de igualdad del tipo idem ac, talis ac, etc.
} 
- Pl., Merc. 897 amicior milhi nullus atque is est ${ }^{29}$;

y hasta por an ${ }^{30}$.

- Sal., Hist. fr. 4, 1 perincertum, stolidior an uanior ${ }^{3 !}$.

Pues bien, las aludidas exigencias de la coordinación consisten en que los términos compartan el mayor número de marcas posibles: tratándose de sustantivos, comparten caso: Caesare ac militibus; si se trata de adjetivos, comparten caso, número y, si lo distinguen, género: mulier docta et formosa; cuando, en fin, el adjetivo presenta grado intensivo, la coordinación no impone el mismo grado en el otro adjetivo, dado que esta sólo puede afectar a marcas sintácticas que garanticen la isofuncionalidad $\rightarrow$ y el grado es marca semántica--, pero, de aparecer la intensidad en él, sí redunda, de paso y por coincidencia, en aquella isofuncionalidad.

Así también se explica muy bien la implícita regla del latín según la cual dos adjetivos coordinados deben ir en el mismo grado ${ }^{32}$ : es lo lógico y lo natural; y que no aparezcan construcciones aberrantes como magis doctus quam prudentior, porque, siendo ahí léxica la indicación del grado, los adjetivos, ambos, quedan libres de expresarla gramaticalmente: magis les afecta por igual; quam los coordina ${ }^{33}$.

Pero también se explican así los no pocos casos en que aquella regla se altera cuando los adjetivos aparecen en distinto grado. En efecto, puede suceder que el segundo adjetivo de la coordinación presente un grado superior al del primero:

-.- Apul., $M$. X 16 gratiosum commendatioremque me ei facere... appetebam;

- B.Alex. 3, 1 homines ingeniosi atque acutissimi;

${ }^{29}$ Otros ejemplos en Kühner--Stegmann, 1914, II, 2, 20. Generalmente aparecen en contextos negativos, aunque, tratándose de adverbios, también se presentan en contextos no negados: Hor.Epod.XV5-6 atrius atque hedera procera adstringitur ilex/lentis adhaerens brachiis...

${ }^{30}$ Correa 1978, p.537, que trae a colación el pasaje de Salustio, no cree, sin embargo, que la construcción sea semejante a doctior quam prudentior. Otro ejemplo, en HofmanñSzantyr 1972, p. 163.

31 La diferencia entre $a c$ y las otras conjunciones parece residir en que, mientras que aquélla une sin valor semántico alguno, éstas unen con valor comparativo, en el caso de quam, y valor interrogativo, en el de an.

32 De hecho, que se señalen pasajes de coordinación en distinto grado como anomalías implica la regla; por ejemplo, en Bassols 1956, p. 168. En los estudios de latín tardío o cristiano se señala también como anomalía; vid., por ejemplo, A. Blaise 1955, p. 100: «Contrairement à l'usage classique, on coordonne des adjectifs ou des adverbes d'un degré différent.»

33. Que no se encuentre la expresión, asimismo aberrante, doctior prudentiore (Correa 1978 , p. 257) se debe a que, en latín, el ablativo se reserva para comparaciones entre sustantivos, no entre adjetivos. 
ahí se cumplen las exigencias sintácticas de la coordinación, pero en los segundos adjetivos hay un valor semántico añadido de elación / superelación que se desea mostrar en contraste con el primero, que carece de él. No hay anomalía alguna ${ }^{34}$.

O puede suceder, al contrario, que ese segundo término presente grado inferior:

- Cat., Agr. III 4 oleum uiridius et bonum;

— Gell., I 4, I doctrina utiliore ac delectabili;

donde una de dos: o los grados están empleados con su valor propio, como opina Correa ${ }^{35}$, o hay en el segundo adjetivo hay una verdadera relajación semántica en la expresión de la elación, en función de la cual aparece un término menos marcado de la oposición en uso neutro por el marcado. Esto último no suele suceder cuando la coordinación se establece con quam, por lo menos hasta Vitrubio ${ }^{36}$, tratándose de adjetivos; cuando son adverbios los coordinados, encontramos algunos ejemplos a partir de Tácito:

- Tac., Hist. I 83 Nimia pietas uestra acrius quam considerate excitauit;

- Tac., Agr. IV 3 uehementius quam caute.

Por lo demás, fuera de coordinaciones de este tipo hallamos algunos usos neutros de positivo por intensivo, como estos, señalados por Correa ${ }^{37}$ :

- Tac., Ann. I 68 quanto inopina tanto maiora;

- Pl., Rud. 1301 quanto magis extergeo, rutilum atque tenuis fit $^{38}$;

Y también de positivo por superlativo:

- Pl., Trin. 1115 Hic homost omnium hominum praecipuos ${ }^{39}$;

donde el grado positivo aparece por el superlativo porque el lexema del adjetivo ya significa de por sí superelación. Eso, junto con el hecho de llevar complementación «inclusiva», la que lógicamente acompaña a un superlativo, permite la relajación de marcas.

34 Así, M. Bassols 1956, 1, p. 168, nota 19: «La coordinación de un positivo y superlativo no implica en la época clásica debilitación de este último, pues en frases de este tipo puede conservar su fuerza intensiva»?.

35 Correa 1978 , p. 538.

36 Vid. Hofmann-Szantyr 1972, p. 163.

37 Correa 1978 , p. 538 , aunque los considere de positivo por «comparativo» (sc. relativo).

38 También de positivo por «comparativo» lo cataloga Arias Abellán 1984, p. 126. Otros ejemplos en Hofmann-Szantyr 1972, p. 170.

${ }^{39}$ Según Arias Abellán, ibid., uso neutro de positivo por superlativo comparativo o relativo. 
Hablemos, por último, de construcciones del tipo optimus quisque, donde el superlativo posee naturalmente valor de elación máxima, pero donde, conforme avaniza la latinidad, tiende a instalarse el intensivo y hasta el positivo. En este último caso, suele ser, como antes, un adjetivo cuyo lexema implica ya superelación ${ }^{40}$ : Lucr. V 1415 pristina quaeque; Liu., 1 7, 5 eximium quemque; Tac., Ann. VI 27, 3 egregium quemque; pero, a veces, también un adjetivo sin idea de superelación: Tac., Ann. XI 43, 1 invalidus quisque. En ambas situaciones podemos hablar de usos neutros del término no elativo por el elativo ${ }^{41}$.

Que aparezca el intensivo, como en Rhet. Her. IV 25, 35 superioris cuiusque... uerbi o en Apul. Mund. 25 propiores quosque, y que sólo en latín tardío sea relativamente productivo el giro ${ }^{42}$, parece achacable, más que a una relajación de marcas o, menos aún, a una «neutralización de la oposición» ${ }^{43}$, a la fijación en esa época de la fórmula «adjetivo + quisque», si bien puede atribuirse originariamente a un uso neutro del término elativo por el superelativo. Al final, cualquier grado acaba apareciendo en el adjetivo; incluso adjetivos que refuerzan la idea de distribución expresada por quisque, completamente innecesarios: singuli quique, donde ya no hay valor alguno de elación.

\section{LA GRADACIÓN EN EL ADVERBIO}

Se sucle admitir que la gradación del adjetivo latino constituye una verdadera categoría gramatical ${ }^{44}$; en cambio, no parece suceder to mismo cuando se examina el fenómeno en el adverbio. En efecto, partiendo de que el adverbio es forma invariable, algunos estudiosos, como H. Pinkster ${ }^{45}$, tratan de explicar su gradación por derivación asimilada a la del adjetivo. Si esto fuera así, en planius/planissime no tendríamos formas de «comparativo» y «superlativo» del adverbio plane, sino adverbializaciones de los grados «comparativo» y «superlativo») correspondientes del adjetivo planus.

El problema de esta interpretación, asumido por Pinkster, estriba en que existen adverbios en grado «comparativo» y «superlativo» que no pueden interpretarse como adverbializaciones de sendas formas de adjetivo, pues en su caso no existen. Así sucede, entre otros, con diutius, diutissime, construidos a

\footnotetext{
40 Hofmann-Szantyr 1972, p. 170.

4 La superelación aquí no cuenta, de la misma manera que en la oposición entre inanimado/animado no cuenta que exista un femcnino, sino sólo que sea animado, con independencia de su género.

42 El empleo de la Rhet.Her: es excepcional. Sólo es más frecuente a partir de Apuleyo y, sobre todo, de Tertuliano, Lactancio y otros (Hofmann-Szantyr 1972, p. 170).

43. Como opina Correa 1978, p. 537.

44 De hecho la categoría se remonta a época indoeuropea; vid. F. R. Adrados 1985, p. 39.

45 Pinkster 1972, p. 70.
} 
partir del adverbio ditu, no de un adjetivo. Bien es cierto, en cambio, que el grado no es aplicable a todo adverbio, ya que muchos no lo conocen.

No obstante, nos parece que el hecho de que los grados del adverbio sean derivados de los correspondientes del adjetivo o se construyan a partir de una forma base de adverbio no afecta para nada a su funcionamiento. En efecto, lo que importa es poder establecer un paradigma de gradación en el que sus términos se opongan según ciertos valores. Y ese paradigma es precisamente lo que, según opinamos, comparten plane/planius/planissime y diu/diutius/diutissime; un idéntico paradigma y unos idénticos valores en sus términos que permiten afirmar que el adverbio posee la categoría de la gradación.

Por otra parte, parece lógico considerar que la noción básica y estructura de la categoría son iguales que en el adjetivo, de la misma forma que la categoría del número, por ejemplo, aunque con diferencias de función, se estructura en torno a la misma noción en el sustantivo y en el adjetivo. Eso explica las semejanzas que en el uso de los grados del adverbio se observan respecto al de los grados del adjetivo; algunas de ellas ya han quedado apuntadas más arriba al tratar de las dobles comparaciones. Las posibles diferencias, en cambio, pueden atribuirse a la distinta naturaleza lingüística de adjetivo y adverbio que implicará, a su vez, distintas relaciones sintagmáticas. Pero ésa ya seria materia de otro trabajo.

\section{CONCLUSIÓN}

No nos atrevemos a dar por zanjado el asunto, y menos aún en lo que afecta al adverbio. Con todo, en nuestro análisis de los hechos nos ha parecido intuir que la categoría de la gradación es una sola, y no dos; que no es su función propia la de comparar - y mucho menos entre dos o más de dos términos-, sino la de expresar elación; y que, a partir de una estructura como la que hemos propuesto, pueden explicarse de una forma más natural y menos traumática los usos de los grados. Ahora, como siempre, la última palabra la tienen los textos.

Pedro Manuel Suárez Martínez

\section{BIBLIOGRAFÍA}

Adrados, F. R. (1985): «Der Ursprung der grammatischen Kategorien des Indoeuropäischen", Grammatische Kategorien. Funktion und Geschichte, Akten der VII. Fachtagung der Indogermanischen Gesellschaft (ed. B. Schlerath), Wiesbaden, pp. $1-46$.

Arias Abellán, C. (1984): «Notas sobre la intensificación del adjetivo en la obra de Plauto), Habis 15, pp. 125-140.

Arias Abellán, C. (1987): «La comparación de inferioridad y la "atenuación del adjetivo" en la obra de Plauto», RSEL 17, pp. 327-339. 
Bassols de Climent, M. (1950-52): «Los grados comparativos», Estudios Clásicos 1, pp. 187-193.

Bassols de Climent, M. (1956): Sintaxis Latina, I, Madrid.

Blaise, A. (1955): Manuel du Latin Chrétien, Estrasburgo.

Correa, J. A. (1978): «Estructura de la gradación adjetiva en latín», Actas del V Congreso Español de Estudios Clásicos, Madrid, pp. 535-539.

Díaz Tejera, A. (1985): «El factor semántico en la configuración categorial. La gradación del adjetivo como ejemplificación», RSEL 15, pp. 277-290.

Ernout, A.-Thomas, F. (1972): Syntaxe Latine, París.

Fugier, H. (1972): «Le système latin des comparatifs et superlatifs», REL 50, pp. 272-294.

Hofmann, J. B.-Szantyr, A. (1972): Lateinische Syntax und Stilistik, Munich.

Kühner, R.-Stegmann, C. (1914): Lateinische Grammatik, II, Darmstadt (= reimpr: Hannover 1982).

Pinkster, H. (1972): On latin adverbs, Amsterdam-Londres.

Poirier, M. (1991): «Superlatif relatif et superlatif absolu. Cette distinction est-elle incontestable?», Comunicación presentada en el VI Colloque International de Linguistique Latine, Budapest, 23-27 de abril.

Ruipérez, M. S. (1954): Estructura del sistema de aspectos y tiempos del verbo griego antiguo. Análisis funcional sincrónico, Salamanca.

Sánchez Salor, E. (1980): «Los dos comparativos latinos», Estudios de Filología Latina en honor de la profesora Carmen Villanueva Rico, Universidad de Granada, pp. $217-226$. 